\title{
Teoria del diritto e punto di vista interno-Sviluppi e ripensamenti ${ }^{*}$
}

\author{
Por MASSIMO LA TORRE \\ Università Magna Graecia di Catanzaro
}

\begin{abstract}
RESUMEN
Lo scritto intende innanzitutto tematizzare la recezione del cosiddetto punto di vista interno nella teoria del diritto contemporanea. Introdotto fondamentalmente da H. L. A. Hart, l'approccio «interno» non ha riscosso tra $i$ giusfilosofi quella fortuna che sembrava in un primo tempo dovergli arridere. Molti degli sviluppi post-hartiani gli sono francamente ostili, a partire da Joseph Raz e dalla sua scuola. Del resto, la prospettiva «interna» (che è quella del partecipe alla pratica giuridica e non dell'osservatore) sembra confliggere con l'impassibilità e neutralità che il giuspositivismo si attende dal teorico del diritto. In Italia tale movimento di ripensamento rispetto al punto di vista interno si manifesta nell'opera ambiziosa e imponente di Luigi Ferrajoli, la cui metodologia «assiomatica» risulta decisamente impraticabile al partecipante ed è solo assumibile dalla posizione di un algido e lontano osservatore e sistematizzatore. Su ciò l'articolo vuole infine soffermarsi.
\end{abstract}

Palabras clave: punto di vista interno, teoria del diritto, Herbert Hart, Ferrajoli, giurisprudencia assiomatica.

* Relazione presentata al Convegno sull'opera Principia juris di Luigi Ferrajoli, Università di Valencia, 26-27 aprile 2012. 


\begin{abstract}
The aim of this paper is to thematize the reception of the so-called internal point of view in contemporary legal theory. The «internal» approach (introduced by H. L. A. Hart) has been collected by legal theorists without the appropriate view in relation to the first analysis. In the post-Hart approach, many developments are frankly hostile, specially those given by Joseph Raz and his school. Moreover, the «internal» prospect (which is the legal practice of the participant and observer) seems to be in conflict with the impassivity and neutrality that for legal positivism is expected from the legal theorists. This movement of rethinking according to the internal point of view, In Italy is manifested by Louis Ferrajoli in his ambitious and impressive work. His "axiomatic» methodology is quite impractical to the participant and only it can be assumed from the position of an icy and distant observer and systematizer; the article will linger on that later.
\end{abstract}

Key words: internal point of view, legal theory, Herbert Hart, Luigi Ferrajoli, axiomatic jurisprudence.

La consapevolezza e la tematizzazione del punto di vista interno è un'acquisizione relativamente recente degli studi giusfilosofici. Nella teoria del diritto, com'è noto, il punto di vista interno è la proposta metodologica avanzata nel libro maggiore di Herbert Hart, Il concetto di diritto. Questa scelta metodologica è ribadita poi nel Poscritto a tale volume, dove si sostiene ancora una volta che il teorico del diritto «deve comprendere ciò che è adottare il punto di vista interno e in questo senso limitato egli deve essere capace di mettersi al posto di un insider» ${ }^{1}$.

In questa prospettiva il punto di vista interno ha una duplice articolazione. (a) La prima è tematizzata da Hart come l'aspetto interno delle norme. Si tratta dell'atteggiamento riflessivo che il destinatario o l'usuario di una norma può assumere rispetto a questa. Egli cioè può usarla come ragione per la propria e l'altrui condotta $\mathrm{e}$ come fondamento di una pretesa o di una critica. In questo senso la norma è inserita in un contesto di discorso e in una pratica nella quale, per essere efficace, per motivare cioè la condotta in questione, dev'essere riconosciuta come ragione valida. Ciò implica che tale validità possa essere contestata, e infine persino negata. La norma non esprime una regolarità, o una mera convergenza di condotte, né una relazione necessaria tra stati di cose; non è la causa o

1 H.L.A. HaRt, The Concept of Law, a cura di P. Bullock e J. Raz, Clarendon, Oxford 1994, 2nd ed., p. 242. Corsivo nel testo. 
il motivo di una condotta, ne fornisce piuttosto la giustificazione, la ragione, proprio in assenza della cogenza del risultato o dell'effetto cui la norma mira.

(b) Il punto di vista interno poi -ed è questa sua seconda dimensione quella propriamente metodologica- è quello della teoria del diritto che per spiegare la pratica e la norma del diritto ripropone e per così dire simula la riflessività del destinatario e dell'usuario del diritto. L' «essenza» del diritto in questa prospettiva rimane la sua pratica, la quale come tale, come pratica, dunque come insieme e pluralità di usi, viene ora resa esplicita dal teorico. Questo deve quindi esercitare una certa astinenza epistemica con riguardo al modo d'operare del destinatario o dell'usuario delle norme giuridiche. Non può sostituire i concetti di questo con delle categorie che gli sono estranee. Semmai potrà procedere in maniera «trascendentale», rintracciando e riconcettualizzando le strutture o -se si vuole- la «mitologia» del discorso e dell'uso giuridico. Ma la categorizzazione di tali strutture e di tale mitologia dev'essere sempre ripresa dalla pratica e presentarsi in modo tale da permettere a questa di riconoscersi nella teoria e di continuare ad essere ciò che è, articolazione di ragioni a favore e di critiche, dunque serie di giustificazioni e di obiezioni, insomma argomentazione e controversia. L'univocità e l'assiomaticità non si prestano a tale compito.

Dal punto di vista interno -come prospettiva metodologia generale- la natura del diritto e la sua struttura devono essere ricostruite e concettualizzate in modo da poter dar conto della controversia e d'assumerla non come patologia della pratica giuridica, bensì come suo tratto definitorio. In particolare, dal punto di vista interno i concetti giuridici - per esempio la nozione di «diritto soggettivo»o quell'altra tanto densa di storia e valori di «costituzione»- non potranno essere svuotati del loro contenuto simbolico-normativo, e isolate dal loro sfondo culturale e valoriale (da ciò che ne costituisce in qualche modo la «precomprensione»), per essere rinchiusi o ritagliati in dispositivi logico-funzionali.

A questo proposito va sottolineato che il diritto si pratica in buona sostanza nella forma di una controversia su diritti contrapposti, la cui rivendicazione implica per ciascuna delle parti la pretesa di essere nel proprio diritto, nel giusto. «A dispetto d'ogni violazione del diritto e d'ogni delusione, ogni conflitto giuridico -ci ricorda Michael Stolleis, eminente storico del diritto tedesco-induce le parti coinvolte a dichiarare la loro posizione ogni volta di nuovo quella "giusta" e i loro avversari ad essere "nel torto" ${ }^{2}$. Ciò è grosso modo quanto Alexy ci rappresenta con la tesi della pretesa di giustizia insita nel discorso giuridico ${ }^{3}$.

2 M. Stolleis, The Eyes of Law, Birkbeck Law Press, New York 2009 p. 70.

Vedi R. Alexy, Begriff und Geltung des Rechts, Alber, München 1990. 
Ciò fa del diritto una pratica controversa ma nello stesso tempo aperta e rivolta (necessariamente) al discorso morale. Questo è ciò che accade nel lato interno del diritto, dal punto di vista interno come aspetto fenomenologico di una pratica secondo norme di diritto. Orbene, una teoria del diritto che di tale aspetto interno si faccia carico non potrà disattivare -a pena di risultare intollerabilmente prescrittiva- la pretesa di giustizia di quella pratica che essa è chiamata a registrare e concettualizzare. La controversia nel lato interno si dà mediante argomenti e rinvii semantici e simbolici che sono strutturali alla pratica in questione. Tale rete di argomenti e rinvii non può essere neutralizzata $\mathrm{o}$-er così dire- normalizzata mediante stipulazioni convenzionali e assiomatiche, decise dal teorico secondo schemi tutti suoi. La voce e i concetti del teorico -sia pure legittimi- non possono mettere a tacere la voce e i concetti della pratica, se non offrendone un'immagine anodina e fondamentalmente distorta.

Dice Stolleis: «Tanto il debole come il forte invocano il diritto. Ciò che questo significa è qualcosa che abbisogna d'essere costantemente ridefinito» ${ }^{4}$. Una tale circostanza -che Stolleis sembra interpretare come una situazione d'autoconvincimento psicologico e di autoaffermazione ideologica- è letta da Ronald Dworkin nei termini della sua «tesi dei diritti», la rights thesis ${ }^{5}$. «Una pretesa di diritto [a claim of $l a w][\ldots]$ è equivalente allora alla pretesa che un principio o un altro [one principle or another] offra una migliore giustificazione di qualche parte della pratica giuridica ${ }^{6}$. Questa tesi può considerarsi come un completamento ed un affinamento della descrizione fenomenologica del lato interno della norma giuridica e della pratica secondo norme di diritto.

Il diritto si usa eminentemente nella controversia su diritti-questo ci dice Dworkin. E la loro rivendicazione, la rivendicazione dei diritti, contiene o implica la pretesa d'aver ragione, una pretesa di giustizia. Qui il lato interno delle norme mette in moto un tipo di giustificazione che fuoriesce dal recinto semantico della norma o regola e che si propone nei termini di un diverso tipo di ragione, ancora più controvertibile e controverso, ma di una dignità normativa più alta della semplice norma. Il lato interno della norma cioè, manifestandosi mediante giustificazioni, obiezioni e critiche ricorre, per sostanziarsi, a riferimenti normativi più forti e universali, e di carattere quindi tendenzialmente morale, i princìpi.

${ }^{4}$ M. StOlleis, Op. cit., p. 74.

5 VeDi R. Dworkin, Taking Rights Seriously, Duckworth, London 1978, capitolo secondo.

${ }^{6}$ R. Dworkin, Justice in Robes, Harvard University Press, Cambridge, Mass. 2006, p. 52. 
Come si è detto, tradizionalmente la teoria del diritto adotta il punto di vista esterno. Ciò è vero, per esempio, per John Austin, il quale pone al centro della sua ricostruzione del concetto di diritto l'idea di sanzione considerata «la chiave della scienza giuridica», «the key to the science of jurisprudence» ${ }^{7}$. D'altra parte, Austin è un fermo sostenitore della definizione per genus et differentiam e non esita ad operare sui concetti giuridici usati dal giurista pratico con un affilato - per così dire- bisturi analitico. Per cui i concetti -come nel caso esemplare del diritto soggettivo- sono sottoposti ad una manipolazione imperativistica e logicistica che ne riduce di molto il campo semantico e la forza normativa e simbolica. Del resto, per Austin programmaticamente i concetti usati nella dottrina e nella pratica del diritto non sono altro -nticipando Alf Ross e il suo «Tû-tû» ${ }^{8}$ - che segni riassuntivi di lunghe serie di proposizioni normative, «short marks for long series of propositions» ${ }^{9}$.

Va inoltre ricordato a questo proposito uno dei punti sui quali con più energia Hans Kelsen si smarca dall' «analytical jurisprudence», e questo è la nozione di Stato. Quella infatti assunta da Austin sembra avere ben poco di giuridico o normativo, giacché dallo studioso britannico lo Stato è concepito essenzialmente come un fenomeno materiale, una situazione di potere di fatto. La sovranità giuridica per Austin si fonda infine e si manifesta nella capacità tutta fattuale di imporre il proprio comando, accompagnata da un abito di subordinazione in chi lo subisce, individuato nuovamente secondo tratti fondamentalmente materiali o comportamentistici ${ }^{10}$.

La teoria di Kelsen però anch'essa non potrebbe -senza perplessità- concepirsi come un contributo offerto dal punto di vista interno, cioè mediante l'assunzione di questo come propria privilegiata prospettiva metodologica. Kelsen, pur differenziando tra causalità e imputazione, sembra pensare quest'ultima spesso nei termini di un'attività stipulativa dell'osservatore o del fruitore della norma. Ciò è dovuto all'ambiguità tanto della sua concezione del Rechtssatz, della cosiddetta proposizione giuridica, che sembra talvolta essere al tempo stesso descrizione della norma e pure norma ${ }^{11}$, quanto della categoria

${ }^{7}$ J. Austin, The Province of Jurisprudence Determined, with an introduction by H. L. A. HART, Weidenfeld \& Nicholson, London 1954, p. 13.

8 Il riferimento ovviamente è a A. Ross, Th-t $\hat{u}$, in «Harvard Law Review», Vol. 79, 1957. p. 371 .

J. Austin, The Uses and the Study of Jurisprudence, in J. Austin, Op. cit.,

${ }_{10} \mathrm{Si}$ veda H. Kelsen, The Pure Theory of Law and Analytical Jurisprudence, in «Harvard Law Review», Vol. 55, 194 pp. 44-70.

${ }^{11}$ A questo proposito indicativo mi pare il seguente passaggio: «I principî logici possono esser applicati a norme giuridiche, se non direttamente, almeno indirettamente, nella misura in cui essi sono applicabili alle proposizioni giuridiche che des- 
messa in campo di norma come «schema d'interpretazione» (Deutungsschema), là dove tale interpretazione sembra essere quella del teorico o dell'osservatore del fatto che soggiace alla relazione normativa.

Kelsen distingue bene ed accuratamente tra atto e significato dell'atto, da cui solo ha origine la norma ${ }^{12}$. Ma egli introduce anche un'altra distinzione, molto plausibile, tra il significato soggettivo e quello oggettivo di un atto. E solo il secondo a darci la norma, non l'ascrizione di senso meramente individuale di un qualche soggetto od organo. Ora però, il significato oggettivo dipende dallo Stufenbau, dal fatto che chi pone in essere l'atto è investito della competenza corrispondente da una norma di gado superiore. Questo sistema gerarchico culmina e si chiude -com'è noto- nella «norma fondamentale», la quale però -ci dice Kelsen- è una presupposizione «trascendentale» dell'osservatore dell'ordinamento in questione. Di maniera che il senso «oggettivo» della norma, cioè il suo senso proprio, finisce per dipendere dall'osservazione esterna e dal metodo di colui che reinterpreta teoricamente il sistema giuridico in cui si dà quella norma come un coerente costrutto di norme disposte gerarchicamente. Questo è un punto di vista gnoseologico che non riflette il punto di vista pragmatico di colui che adopera la norma in questione. E sì per certi versi ermeneutico, giacché distingue tra fatto e senso del fatto; ma rimane ancora del tutto esterno alla pratica entro la quale si dà la norma cui si ascrive il significato «oggettivo», poiché il senso del fatto è qui affare di chi descrive il sistema, non di chi opera all'interno d'esso e con esso.

«La società è [...] -dice Kelsen- un oggetto completamente diverso dalla natura» ${ }^{13}$. Ed il diritto è riconosciuto come un «fenomeno sociale». Ciò che distingue la società dalla natura è il «significato» che si ascrive a certi atti empirici o psicologici, e tuttavia la sociologia del diritto che studia la società come proprio oggetto specifico d'indagine si occupa -dice Kelsen- di fatti naturali. «Questa sociologia del diritto non pone in relazione $i$ fatti naturali che deve studiare con le norme valide, ma stabilisce un rapporto di causa ed effetto fra questi e altri fatti naturali» ${ }^{14}$; il che ha come conseguenza che la sociologia del diritto non si occupa invero del diritto, bensì di«certi fenomeni paralleli della natura ${ }^{15}$. È la scienza giuridica la scienza propria

crivono queste norme giuridiche» (H. KELSEN, La dottrina pura del diritto, a cura di M. G. Losano, Einaudi, Torino 1966, p. 91). Qui sembra che il criterio di verità o falsità che vige per la proposizione giuridica si possa (seppure indirettamente) trasmettere alle stesse norme giuridiche; suggerimento abbastanza ambiguo, ma rivelatore di una latente sovrapposizione della norma e della proposizione che verte su di essa. del 1934

i2 Si legga per esempio il capitolo primo della Reine Rechtslehre, I edizione,

13 H. Kelsen, Lineamenti di dottrina pura del diritto, trad it. di R. Treves della I ed. della Reine Rechtslehre, Einaudi, Torino 1973, p. 48.

14 Ivi, p. 54.

15 Ibidem. 
del diritto, giacchè è essa -e solo essa- che verte e si intrattiene sul «significato» che costituisce il fenomeno del diritto.

Non pare riconosciuta la vigenza di norme che siano tali pur non essendo giuridiche. Solo queste ultime sembrebbe che mantengano un significato «oggettivo» e dunque normativo in senso proprio. Le altre norme, quelle morali per esempio, -in assenza di una loro incardinazione nello Stufenbau e della corrispondente opera gnoseologica dello scienziato giuridico- rimarrebbero confinate alla sfera del significato «soggettivo», il quale però si mantiene come qualcosa di fondamentalmente psicologico, dunque permane un fatto naturale.

D'altra parte, per Kelsen -come per Austin- è la sanzione ciò che definisce la norma come giuridica e la sanzione è tale perché fatto materiale indipendente da una previa qualificazione normativa. $\mathrm{Su}$ questo punto, com'è noto, si esercitano la critica di Hart, e quella -mi piace ricordare- di Ota Weinberger ${ }^{16}$, che notano come la sanzione non possa distinguersi da altre conseguenze negative per gli interessi del consociati (per esempio il pagamento delle tasse) in assenza di una sua specifica qualificazione giuridica.

Inoltre per Kelsen il senso (o il dover essere) della norma è qualcosa di riconoscibile o imputabile -quando non lo sia (anche) al teoricoeminentemente al soggetto o all'organo che pone la norma, paradigmaticamente al legislatore (sia questo anche costituente o meno). Colui al quale la norma si dirige per regolarne la condotta è concettualizzato o in termini passivi come destinatario oppure come nuovo livello di produzione discrezionale di senso normativo. La teoria si fissa dunque sul momento e sulla fenomenologia della produzione della norma (sia pure a distinti livelli di generalità) intendendo la norma come attività fondamentalmente decisoria, imperativa. L'uso della norma secondo una diversa modalità -quello per esempio rivendicativo o argomentativo dell'avvocato o del semplice cittadino- non è tematizzato.

Da qui l'insufficiente e la generale implausibilità della sua teoria dell'interpretazione, per la quale questa dovrebbe limitarsi ad una lista aperta di possibili soluzioni ermeneutiche senza l'indicazione della risposta considerata corretta ${ }^{17}$. Concezione dell'interpretazione questa che non rispecchia la pratica effettiva del giurista in azione e nemmeno quella più comune del giurista cosiddetto dogmatico. Così come traducendo si offre del linguaggio tradotto una versione che si propone come quella migliore possibile (vale a dire la migliore di cui si è capaci in un certo momento) così della norma l'interpretazione tende

16 Vedi H. L. A. Hart, The Concept of Law, cit., p. 39, e O. Weinberger, Der Sanktionsbegriff und die pragmatische Auswirkung gesellschaftlicher Normen, in Normenlogik, a cura di H. LenK, Verlag Dokumentation, Pullach bei München, 1974, pp. 89-111.

17 Si veda per esempio H. Kelsen, Reine Rechtslehre, ristampa della I ed. del 1934, a cura di M. Jestaedt, Mohr, Tübingen 2008, pp. 100 ss. 
ad indicare per la questione cui si applica la risposta corretta (anche qui ovviamente sempre sulla base dell'informazione e delle conoscenze di cui al momento si dispone).

La teoria dell'interpretazione di Kelsen accusa dunque una notevole distanza dalla pratica del diritto e dai suoi usi concreti. Per non parlare poi dell'adesione del giurista austriaco alla teoria dell' «ideologia» di provenienza marxista. Secondo Kelsen buona parte del discorso interno del giurista è imbevuto - per così dire -di pregiudizi e finzioni meramente «ideologiche», cioè sovrastrutturali. Queste servono ad occultare la vera realtà del diritto- che sarà quella che ci indica ora il teorico capace di penetrare la cappa oscura e confusa della «sovrastruttura» per giungere a toccare e vedere la «struttura» delle cose. Una posizione questa che ricorda da vicino quell'altra dei realisti scandinavi, di Axel Hägerström o di Karl Olivecrona, che accusavano il discorso dei giuristi d'essere fondamentalmente simile a quello della «magia», pieno zeppo di concetti e termini senza referenti reali nel mondo empirico, pura fantasia. "Abbiamo l'illusione -dice Olivecrona- che la parola "diritto soggettivo" significhi un potere su un oggetto, sebbene si tratti di un potere che non possiamo mai capire ${ }^{18}$. I giuristi insomma come una tribù primitiva, agitata da miti e riti senza alcun fondamento di verità.

Il trattamento che Kelsen dà della questione intricata e anche spinosa e politicamente sensibilissima del diritto soggettivo -ch'egli vuole a tappe forzate ricondurre al diritto oggettivo, facendone solo una specie del diritto in senso soggettivo al pari dell'obbligo- è a questo proposito una mossa particolarmente significativa e radicale, segnalando ancora una volta la distanza della teoria dalla complessa pratica dell'uso di norme così come essa si realizza nell'attività del giurista pratico e del comune cittadino.

Oppure si pensi ancora alla maniera idiosincratica -che dipende nuovamente dalla centralità concessa al fatto della sanzione- di concettualizzare la norma giuridica come una regola che non definisce ciò che è illecito o scorretto fare ${ }^{19}$. Di maniera che il diritto non ci direbbe o prescriverebbe ciò che è giusto fare, non dirigerebbe la condotta dei consociati, ma si limiterebbe soltanto a stabilire delle condizioni o fattispecie alle quali conseguono e s'impongono delle sanzioni. L'illecito non sarebbe allora una violazione del diritto, ma piuttosto ciò che ne conferma la vigenza, mettendolo in moto: «Il diritto non può essere infranto o violato dall'illecito perché è anzi soltanto a mezzo suo che il diritto raggiunge la propria essenziale funzione» ${ }^{20}$. Il diritto pertanto dirigerebbe l'azione dei cittadini in modo obliquo, allusivo, per certi versi subdolo. La norma che Kelsen chiama «secon-

18 K. Olivecrona, Law as Fact, II ed., Stevens \& Sons, London 1971, p. 184.

19 Punto questo su cui si esercita l'acuta critica di Hart (vedi The Concept of Law, cit., p. 39).

${ }_{20}$ H. Kelsen, Lineamenti di dottrina pura del diritto, cit., p. 68. 
daria»-quella che si dirige alla condotta dei cittadini e degli altri soggetti giuridici- sarebbe il risultato di un'inferenza logica a contrario. La quale tra l'altro presuppone una certa operatività della logica in àmbito normativo, operatività che -come sappiamo-il Kelsen più maturo sempre di più tende a mettere in discussione per poi infine negare con decisione.

È pur vero che per Kelsen la teoria del diritto ha come oggetto privilegiato la norma e le relazioni normative, ma queste sono tematizzate non come quelle che ritroviamo nella pratica giuridica di giuristi e cittadini, bensì come un concetto o una trama di concetti la cui definizione e fenomenologica ci è offerta dal teorico stesso. Il punto di vista rimane qui del tutto esterno, nonostante il conclamato normativismo delle tesi sostenute. Il punto di vista interno infatti non consiste di uno studio che abbia per oggetto norme e concetti normativi, o che usi una nozione tutta teorica e stipulativa di norma come categoria esplicativa, bensì di uno studio che vertendo su norme e concetti normativi assuma questi stessi come schemi interpretativi del proprio studio.

\section{III}

La teoria pura di Kelsen rimane dunque - può ritenersi- ancora esterna al punto di vista interno. La svolta si dà solo e veramente con l'opera di Herbert Hart.

Il giusfilosofo britannico muove innanzitutto da una presa di distanza dal positivismo logico alla maniera del Circolo di Vienna, alla maniera di Schlick o di Carnap. È piuttosto il «secondo» Wittgenstein per lui il filosofo di riferimento. La critica di Hart si appunta in ispecie sulla semantica riduzionistica che comprime in maniera drammatica la complessità e la significanza degli usi del linguaggio ordinario. Hart in particolare non approva la centralità attribuita agli enunciati delle scienze empiriche ed in subordine al linguaggio formale della logica. «E stato un errore accecante di molta filosofia del passato -scrive nell'introduzione agli Essays of Jurisprudence and Philosophy del 1983-, e più di recente de positivismo logico degli anni d'anteguerra, assumere che ci siano solo poche forme di discorso dotato di senso (il discorso della "descrizione di fatti" empirica, oppure l'enunciazione di verità stipulative o logicamente necessarie) e di scartare come privi di senso o semmai come espressioni di sentimento tutti gli altri usi del linguaggio» ${ }^{21}$.

Ciò gli fa prendere anche le distanze da ogni tentativo di applicare la metodologia in uso per le scienze empiriche alla scienza giuridica ed alla teoria del diritto. Per la comprensione di ciò che è diritto -leg-

${ }^{21}$ H. L. A. Hart, Essays in Jurisprudence and Philosophy, Clarendon, Oxford 1983, pp. 2-3. 
giamo sempre nella introduzione agli Essays of Jurisprudence and Philosophy - «la metodologia delle scienze empiriche è inutilizzabile; ciò di cui c'è bisogno è un metodo "ermeneutico" che consista nella descrizione della condotta governata da norme così come essa appare ai partecipanti ad essa, i quali la considerano come l'osservanza o la mancanza d'osservanza a certi criteri condivisi» ${ }^{22}$.

La chiave del concetto di diritto -si dice - è «la distinzione tra regole sociali e la semplice abitudine convergente di comportamenti» ${ }^{23}$. «Questa distinzione è cruciale per la comprensione del diritto» ${ }^{24}$. Basti solo considerare la fenomenologia dell'attività giudiziaria: il giudice «nel punire prende la norma come la sua guida e la violazione della norma come la ragione e la giustificazione per punire chi pone in atto l'offesa» ${ }^{25}$. Il suo punto di vista non è quello di uno spettatore, d'un osservatore per il quale la norma giuridica diventa l'indicazione di una probabilità di condotta.

La presa in carico del punto di vista interno deve riflettersi al livello della teoria del diritto, di modo che gli enunciati di questa non risultino estremamente esterni come quelli di uno spettatore od osservatore, bensì «dall' esterno facciano riferimento alla maniera in cui essi si occupano di quelli dal punto di vista interno» ${ }^{26}$.

Ciò si riconnette all'impossibilità di dar conto della natura del diritto come una «essenza» ed alla difficoltà d'offrire di tale natura una definizione per genus et differentiam. Anche perché -dice Hart nel primo capitolo del Concetto di diritto-semmai è la definizione secondo il «focal case», o «caso paradigmatico», quella che più si addice al diritto. Il cui concetto rimane -sottolinea il giusfilosofo britannico- fondamentalmente controverso. Allora - dice- «il miglior corso d'azione è quello di differire la risposta alla domanda "cos'è diritto" fino a che non si sia trovato ciò che intorno al diritto rende perplessi coloro che hanno posto la questione o hanno provato a rispondere ad essa, anche se la loro dimestichezza col diritto e la loro abilità nell'identificare campioni di questo sono fuori questione» ${ }^{27}$.

22 Ivi, p. 13.

23 H. L. A. Hart, The Concept of Law, cit., p. 12.

24 Ibid.

25 Ivi, p. 11, corsivo nel testo.

26 Ivi, p. 89. Per questa tesi -che equivale alla teorizzazione per l'appunto del «punto di vista interno»- il riferimento immediato di Hart è quanto esposto e proposto nell'opera di Peter Winch, The Idea of Social Science, Routledge \& Kegan Paul, London 1958, testo che è espressamente citato in The Concept of Law (vedi ivi, pp. 289-297). La distinzione dei due punti di vista si trova già comunque in nuce formulata in L. Wittgenstein, Philosophische Untersuchungen, Suhrkamp, Frankfurt am Main 1977, § 82, p. 66, allorché si distingue tra due modi di concettualizzare la regola di una condotta: (a) l'ipotesi esplicativa di un osservatore, oppure (b) la regola che chi pone in essere la condotta ci darebbe come giustificazione della sua condotta, se dovessimo chiedergliene conto.

27 H. L. A. HART, The Concept of Law, cit., p. 15. 
Dopo Hart la teoria del diritto si è ritrovata dunque a doversi confrontare con la tematizzazione del punto di vista interno come un momento centrale della sua riflessione metodologica. Ora, tale confronto ha dato luogo a risultati assai diversi e talvolta contrapposti.

Si possono identificare almeno tre linee direttrici di riflessione ed evoluzione. Da un lato per certi versi la radicalizzazione del punto di vista interno e il suo ricollegarsi ad un'esplicita teoria normativa forte -com'è il caso della proposta di Ronald Dworkin- che fa saltare la neutralità avalorativa del teorico e spinge per l'abbandono complessivo del positivismo giuridico. C'è poi il tentativo di mantenersi fedeli al disegno originario di Hart (arricchendolo semmai con una elaborata teoria del ragionamento giuridico e con un'incursione nel terreno dell'ontologia (per esempio mediante la categoria di «fatto istituzionale») - com'è il caso della ricerca di Neil MacCormick ${ }^{28}$.

E c'è poi quello che chiamerei il «ripensamento» rispetto al rilievo attribuito al punto di vista interno, che qui ora viene rivisto da una prospettiva dai marcati tratti essenzialistici. Questo «ripensamento» mi pare particolarmente evidente nell'opera di Joseph Raz, discepolo di Hart, e nella sua versione di positivismo giuridico «esclusivo», di un positivismo cioè che nega ogni connessione -sia pure eventualetra diritto e morale, almeno per ciò che concerne la questione della «natura» del diritto.

A tal fine Raz distingue nettamente tra teoria della «natura» del diritto, che sarebbe impresa squisitamente filosofica, e che può aver luogo senza dover mettere in campo alcuna nozione normativa forte $o$ morale, e teoria del ragionamento giuridico, o dei giuristi, la quale ammette Raz- non può fare a meno di qualche riferimento a criteri etici o morali. La teoria della «natura» del diritto è un'impresa diretta a conoscere ciò che è l'essenza del diritto, al di là o al di qua della sua pratica contingente e dei suoi caratteri accidentali.

Così, «la teoria del diritto -dice Raz- è lo studio delle proprietà essenziali del diritto» ${ }^{29}$. "Come ho detto - continua-, ci sono caratteri essenziali del diritto, ma il possesso del concetto del diritto non è esso stesso necessario in tutte le culture che hanno sistemi giuridici» ${ }^{30}$.

Questa posizione è attribuita da Raz allo stesso Hart. È vero -argomenta- che Hart sostiene che si seguono delle norme, se si è in grado di riconoscerle come tali, ma ciò non comporta ancora che esse siano riconosciute e concettualizzate come norme del diritto. Il possesso e

28 Di MACCoRMick programmatico è il libro Legal Reasoning and Legal Theory, Oxford University Press, Oxford 1978, dove nell'appendice (On the Internal Aspect of Norms) troviamo un'acuta rielaborazione del punto di vista interno. Si veda anche N. MacCormick, H. L. A. HART, Arnold, London 1981, dove è reso esplicito e recepito criticamente il legato di Hart -che viene altresì etichettato come «ermeneutico» (vedi per esempio, ivi, pp. 37-38).

${ }_{29}$ J. Raz, Between Authority and Interpretation, Oxford University Press, Oxford 2009, p. 90.

30 Ivi, p. 98. 
l'uso del concetto di diritto è logicamente indipendente dal fatto che si viva in una comunità retta secondo il diritto. «Sembrerebbe -continua- che Hart non s'impegni alla tesi secondo cui per vivere in una società governata dal diritto ci sia bisogno d'essere consapevoli del concetto di diritto, al di là della consapevolezza delle norme che di fatto costituiscono il diritto della società medesima».

È possibile -ci dice Raz-seguire delle norme di diritto ed essere consapevoli di seguirle senza sapere che si tratti di norme di diritto, senza cioè capire il loro «succo», senza riconoscere o intuire il loro senso o significato simbolico e morale, e comunque senza avere un'idea o un concetto di ciò che è il diritto. Il che in altri termini sarebbe come affermare che si può giocare un gioco, ovvero seguirne le regole, senza sapere che si tratta di un gioco e senza avere la nozione di gioco.

Dimodoché, -conclude Raz- «il nostro concetto di diritto non fa della consapevolezza d'esso una precondizione del fatto che quella società sia governata dal diritto» ${ }^{31}$. Quando Raz qui dice il «nostro» concetto di diritto, sta parlando di sé ma anche di Hart. Ciò però è una forzatura, la quale si rende manifesta, allorché aggiunge che «il concetto di diritto non è riflessivo, cioè si applica a pratiche sociali di società nelle quali il concetto stesso non è né usato né conosciuto» ${ }^{32}$ -tesi che urta frontalmente con l'idea hartiana della natura del diritto come concetto ermeneutico. Ma ancora di più forse tale forzatura si rende evidente, allorché Raz per giustificare la sua impostazione distingue tra la «natura» del diritto e la sua «esemplificazione», il suo manifestarsi in una pratica o in un ordinamento concreto.

I fatti sociali -scrive Raz- «non determinano la natura del diritto, ma solo la loro esemplificazione» ${ }^{33}$. Questa però è una tesi che confligge con la centralità concessa da Hart nel lavoro definitorio del teorico al «caso esemplare». Essa entra in rotta di collisione col rigetto da parte di Hart delle definizioni essenzialistiche e con la sua adesione al programma di ricerca del «secondo» Wittgenstein. Per questo -come è noto- sono gli usi e le pratiche a fornirci la pista per giungere ai concetti, soprattutto là dove si tratti di usi e pratiche sociali, cioè riflessive, caratterizzate da ciò che Hart chiama «reflective attitude» ${ }^{34}$. Capiamo la regola in primo luogo con riferimento ad un esempio di sua applicazione ${ }^{35}$ che può giudicarsi corretto o sbagliato, e poi rivolgendoci al suo «senso», che è da intendersi nell'orizzonte di una pra-

31 Ivi, p. 94.

32 Ivi, p. 99.

33 Ivi, p. 92.

${ }_{34}$ Vedi H. L. A. HarT, The Concept of Law, cit., p. 57.

35 Cfr. L. WitTGensteIn, Della certezza, trad. it. di M. Trinchero, Einaudi, Torino 1978 , p. 26 : «Per stabilire una prassi non sono sufficienti le regole, ma abbiamo bisogno anche di esempi. Le nostre regole lasciano aperte certe scappatoie. E la prassi deve parlare per se stessa». 
tica collettiva, il «gioco» di Wittgenstein ${ }^{36}$. Questo a sua volta assume ai nostri occhi il suo significato complessivo -il «Witz» di Wittgenstein $^{37}$ - sulla sfondo di una «forma di vita». «Ciò che gli esseri umani fanno valere come giustificazione - dice ancora Wittgenstein- mostra come pensano e come vivono» ${ }^{38}$.

Il concetto di diritto di Hart -come si è visto, ce lo dice lui stessosi fa carico della riflessività delle pratiche sociali, presentandosi esso stesso come riflessivo, «ermeneutico». Non è dunque né meramente empirico né archimedicamente teorico o filosofico. Del resto ce lo conferma Raz, allorché questo afferma che secondo Hart «fa parte della natura del diritto che la sua esistenza sia nota a coloro che vi sono soggetti» ${ }^{39}$, situazione che comporta abbastanza plausibilmente che coloro che sono soggetti al diritto e sono consapevoli d'esserlo siano anche in possesso del concetto o dell' «idea» corrispondente, del concetto vale a dire di quella «cosa» di cui riconoscono la realtà.

L'opera di Raz sembra comunque segnare una sorta di controriforma essenzialistica e l'abbandono del punto di vista interno come prospettiva filosofica ${ }^{40}$. Ciò è in parte comune anche al giusnaturalismo di John Finnis che reinterpreta la nozione di caso paradigmatico proposta da Hart nei termini di una sorta di forma platonica alla quale è intrinseca la dimensione della bontà ed anzi dell'eccellenza. Il «tipo ideale» di Max Weber viene ulteriormente idealizzato nella direzione di un' essenza cui inerisce un principio di perfezione. Il «caso paradigmatico» di Hart -in questa prospettiva- segnalerebbe un'eccellenza morale di cui le diverse esemplificazioni fattuali potrebbero essere dei campioni difettosi o delle corruzioni ${ }^{41}$. Qui l'essenzialismo -a differenza di quanto avviene nell'opera di Raz- assume una netta connotazione eticistica. Questa sarà tale da offrirci delle direttive etiche sos-

\footnotetext{
36 Vedi ivi, p. 84: «Un giuoco linguistico è una cosa che consiste di azioni del giuoco ripetute nel tempo».

37 Sulla nozione di «Witz» si leggano i paragrafi 564 e 567 di L. WiTtGensteIN, Philosophische Untersuchungen, Suhrkamp, cit, § 564 e § 567, pp. 237-238.

38 «Was die Menschen als Rechtfertigung gelten lassen-zeigt, wie sie denken und leben» (Philosophische Untersuchungen, cit., § 325, p. 167). Si legga anche ivi, § 242, p. 139, dove si rinvia esplicitamente alla «forma di vita», Lebensform, come fondamento del giudizio di correttezza.

39 J. RAZ, Between Law and Interpretation, cit., p. 93.

40 A RAz, nondimeno, dobbiamo un'acuta rielaborazione del punto di vista interno, allorché egli, analizzando gli «enunciati normativi» (attraverso i quali si esprimerebbe il punto di vista interno), distingue tra enunciati «committed», «impegnati», e «non-committed» (ovvero «detached»), «disimpegnati» o «distaccati» (vedi J. RAZ, The Concept of Legal System, II ed., Clarendon, Oxford, 1980, pp. 234 ss.). Questa distinzione è ripresa ma diversamente articolata da MacCormick che distingue il punto di vista interno in «cognitively internal» e «volitionally internal» (vedi N. MacCormick, Legal Theory and Legal Reasoning, cit., pp. 275 ss.).

${ }_{41} \mathrm{Si}$ veda J. FInNIS, Natural Law and Natural Rights, Oxford University Press, Oxford 1980, capitolo primo.
} 
tanziali di ciò che sarebbe l'eccellente, il perfetto, e dunque la pienamente e paradigmaticamente realizzata natura del diritto ${ }^{42}$.

Il punto di vista interno dunque risulta ostico tanto al giuspositivismo esclusivo di Raz quanto al giusnaturalismo similmente esclusivo (ma in una contraria direzione, in quella di credere di poter fare a meno della deliberazione come passaggio epistemico della produzione diritto) che mi pare proprio di Finnis. Ma non è che il punto di vista interno goda di troppa buona salute -se così mi posso esprimere- in altre regioni della contemporanea teoria del diritto e nemmeno -mi pare d'intravvederenell'imponente disegno sistematico di Luigi Ferrajoli.

\section{IV}

Il modello metodologico cui si ispira l'opera giusfilosofica del professor Ferrajoli è quello del linguaggio «teorico», in quanto distinto dal linguaggio «osservativo»e «protocollare» di Rudolf Carnap ${ }^{43}$. Contro la proposta di Carnap, com'è noto, si sono fatte valere una batteria di obiezioni a partire da quelle di Otto Neurath ${ }^{44}$ che Ferrajoli riporta e dà per giustificate. Neurath riteneva che fosse impossibile un linguaggio protocollare puro per il fatto che gli enunciati di un tale linguaggio dovrebbero sempre rimandare ad altri enunciati ed esperienze. Il linguaggio protocollare (osservativo) non potrebbe cioè sfuggire a certo olismo, alla presupposizione di asserzioni non ancora verificate o esperite.

Ferrajoli dà per buona la critica di Neurath, ma poi aggiunge che il modello protocollare di Carnap si giustifica invece per ciò che concerne lo studio e l'analisi del diritto. Qui la dogmatica giuridica sì che instanzierebbe un caso di linguaggio osservativo protocollare, su cui opererebbe con strumenti formali la teoria del diritto. «La lettura delle norme -scrive Ferrajoli in un saggio del 1983-e la loro immediata riformulazione concettuale ("l'articolo x della legge $\mathrm{z}$ dice che '...") $[\ldots]$ hanno il carattere di quelle che il primo neopositivismo chiamava "enunciati protocollari" o "protocolli" o "asserzioni di base"» ${ }^{45}$.

42 Di maniera che per FinNis il punto di vista interno rilevante è quello dell'uomo serio e virtuoso, dell'aristotelico «spoudaios», «the mature man of practical reasonableness» (J. Finnis, Op. ult. cit., p. 15).

${ }^{43}$ La proposta metodologica di Ferrajoli è presentata nel dettaglio nel suo Principia juris. Teoria del diritto e della democrazia, Vol. 1, Teoria del diritto, Laterza, Roma-Bari 2007.

44 Vedi O. Neurath, Soziologie im Physikalismus, in «Erkenntnis», Vol. 2, 1931-1932, pp. 393-431, e O. Neurath, Protokollsätze, in «Erkenntnis», Vol. 3, 1932-1933, pp. 204-214.

45 L. FerRajoli, La semantica della teoria del diritto, in La teoria generale del diritto. Problemi e tendenze attuali, a cura di U. Scarpelli, Comunità, Milano 1983, p. 101. 
Su questo punto, però, sorge immediatamente una perplessità: il linguaggio osservativo o protocollare nel modello di Carnap si dà come registrazione individuale (addirittura con nome e cognome del protocollante) d'esperienze sensibili oppure in termini fisicalistici. Si tratta cioè d'un linguaggio che concerne sensazioni empiriche e fatti fisici nel mondo. Lo stesso non potrà dirsi -mi pare- del linguaggio della dogmatica giuridica, al di là del fatto, in tale contesto abbastanza banale, che ogni linguaggio ha una dimensione empirica, tale da essere percepibile ai sensi come fenomeno ottico o uditivo oppure tattile. $\mathrm{Al}$ di là di questa inevitabile soglia empirica -che vale evidentemente anche per il linguaggio religioso o artistico- difficilmente potrà sostenersi che la dogmatica giuridica sia un linguaggio osservativo e protocollare. Tranne che non si voglia limitarla alla registrazione dell'emissione di suoni o segni, senza porsi la questione del significato di questi.

Tanto meno potrà plausibilmente affermarsi che la dogmatica sia un linguaggio fisicalista, vale a dire più o meno immediatamente traducibile nel linguaggio della scienza fisica, secondo le indicazioni di Neurath e di Carnap. Questi -com'è noto- in tal maniera miravano a realizzare l'ideale dell'unità delle scienze secondo una lingua enciclopedica comune, sotto l'egida di quella che a loro avviso rappresenta la regina delle discipline scientifiche, la fisica per l'appunto. Sono noti a questo proposito gli argomenti di Carnap in favore di una ristrutturazione, per esempio, della psicologia nei termini del vocabolario della fisica $^{46}$.

Ora, gli enunciati della dogmatica giuridica consistono piuttosto d'interpretazioni, non di osservazioni; interpretazioni, le quali vertono su complesse entità semantiche e simboliche nelle quali un ruolo determinante è svolto da termini e concetti non riducibili a meri agglomerati di enunciati normativi od alla registrazione di relazioni deontiche. La teoria del diritto, data pure per buona la sua netta separazione dalla dogmatica, non avrebbe allora un primitivo materiale osservativo, registrazioni empiriche di base, su cui lavorare sistematicamente, bensì reti di significati e di concetti simbolici e culturali articolati stoicamente e intrisi di elementi valorativi. Per cui le operazioni sintattiche poste in essere dalla teoria, operando mediante stipulazioni e un sistema finito di termini primitivi e di postulati su un tessuto semantico complesso e tutt'altro che atomico o semplice, avrebbe come risultato un'aggressiva destrutturazione e ricomposizione di significati già consolidati e istituzionalizzati veicolati dalla dogmatica e dalla stessa legislazione e da entrambe messe in circolo nella pratica effettiva di giuristi e cittadini. Ciò ovviamente non impedisce che le concezioni della dogmatica non si possano -diciamo così-sfidare e riformare; una tale impresa potrà compiersi o dall'interno del discorso giuridico

${ }^{46}$ Esemplare in merito è il saggio di R. CARnaP, Die physikalische Sprache als Universalsprache der Wissensschaft, in «Erkenntnis», Vol. 2, 1931, pp. 432-465. 
stesso oppure dal punto di vista ultraesterno della critica morale e politica.

È pur vero che Ferrajoli ammette il carattere ermeneutico degli enunciati della cosiddetta scienza giuridica, ma questo loro carattere non è ulteriormente tematizzato. Ferrajoli invece subito aggiunge che esso non osterebbe a concepire la scienza giuridica, la dogmatica cioè, ancora una volta come scienza empirica, poiché -afferma- le generalizzazioni della dogmatica possono configurarsi come il frutto di ciò che i logici chiamano «induzione perfetta», vale a dire una induzione di ipotesi a partire da un numero limitato di esempi. L'enunciato ermeneutico della dogmatica è così ricondotto alla sostanza di un enunciato induttivo, e il senso su cui esso si esercita alla probabilità di un certo evento fisico. Una tesi questa che ricorda la riformulazione della questione del senso e della norma nei termini della chance di un certo risultato, proposta da Max Weber ed aspramente criticata da Hans Kelsen ${ }^{47}$.

Insomma, ciò che propone Ferrajoli è di riconcettualizzare la dogmatica giuridica come scienza osservativa, vale a dire come osservazione che verte su norme. "I "fatti osservati" dalla teoria e più in generale dalla scienza del diritto -dice- sono le norme giuridiche» ${ }^{48}$. E nondimeno la scienza giuridica manterrebbe, a suo avviso, un carattere ermeneutico. Ma -vale qui sottolineare- l'attività ermeneutica, che è innanzitutto comprensione di un senso, è un'operazione epistemica ben diversa dalla registrazione e descrizione di un dato empirico. D'altra parte, in cosa può consistere l' «osservazione di norme» di cui dovrebbe farsi carico la dogmatica, se non nella comprensione e interpretazione del senso delle norme medesime, giacché le norme come tali si manifestano a noi, ed esistono per noi, solo nel loro senso? Una tale comprensione ed interpretazione non potrebbe tra l'altro essere operazionalizzata secondo un calcolo matematico. Il significato non è né un'entità spazio-temporale, né una quantità numerica. Orbene, l'osservazione di cui sono portatori gli enunciati protocollari può però vertere e darsi eminentemente con riferimento a queste due specie di entità.

Invero, 1' «osservazione» come operazione epistemica non si addice ad una norma; sì però ad un fatto empirico causalmente determinato. E non si addice alla norma, perché questa si esperisce non mediante registrazione visiva o sensoriale, bensì -vale ripeterlo- mediante comprensione, giudizio di senso, ed esiste solo dal punto di vista di colui che, comprendendola, potrebbe allora adoperarla -dal punto di vista cioè non dell'osservatore esterno, ma da quello del partecipante interno alla pratica di senso. L'osservazione di una norma è dunque

47 Si legga H. KELSEN, Der soziologische und der juristische Staatsbegriff, II ed., Mohr, Tübingen 1928, pp. 156 ss.

${ }^{48}$ L. Ferrajoli, La semantica della teoria del diritto, cit., p. 84, corsivo nel testo. 
una locuzione che, intesa come operazione conoscitiva, si presta ad essere etichettata come un ossimoro. Sarebbe come dire stipulazione di un fatto, o interpretazione di un sasso, sebbene in direzione contraria. Comunque, se pure se ne accettasse la significatività, l' «osservazione delle norme» significherebbe una sorta di presa in carico delle norme soltanto dal loro solo aspetto esterno, quello di qualche fatto più o meno rilevante, senza più riguardo al loro aspetto interno, che è quello che ci rivela veramente cos'è la norma in questione, cioè ciò che essa ci dice e ci richiede o ci offre, ciò il suo significato, oltreché il senso che ha dentro la pratica nella quale si dà, quello che si è chiamato già il suo «succo».

L'interpretazione o l'analisi del linguaggio nella scienza giuridica -ripete Ferrajoli- svolge lo stesso ruolo dell'osservazione sperimentale nelle scienze naturali. Il linguaggio della teoria del diritto invece, mancando di riferimento diretto al discorso normativo, è un linguaggio artificiale costruito dal teorico e come tale formalizzabile secondo una sintassi rigorosamente predeterminata. In questa prospettiva le tesi della dogmatica potrebbero configurarsi come proposizioni suscettibili d'essere considerate come giuridicamente vere o false con riferimento alle norme su cui vertono, tanto più che i concetti che una tale scienza, la «dogmatica», utilizza vengono considerati «nomi metalinguistici di norme».

Diverso -ci dice Ferrajoli- è lo statuto semantico dei concetti usati dalla teoria del diritto- di cui «norma» e «diritto soggettivo» sono esempi paradigmatici. Per inciso, se «norma» è un concetto proprio della teoria del diritto, forse ci si potrà domandare come farà la scienza giuridica del dogmatico ad «osservare» norme come fatti empirici mediante enunciati protocollari, posto che «norma» sembra ora essere un concetto stipulativo e convenzionale di cui il dogmatico ancora non dispone. Il dogmatico per osservare norme dovrebbe già essere in possesso di una qualche sua nozione di norma.

Nel caso della teoria del diritto si tratta -ci dice ancora Ferrajolidi termini che mancano di una relazione semantica diretta con la realtà del diritto, sia che questa la si studi dal di dentro come norma ovvero dal di fuori come fatto. La norma qui sembrerebbe allora essere qualcosa che è allo stesso tempo oggetto d'osservazione empirica come fatto (dal di fuori o dal punto di vista esterno) e di interpretazione come entità semantica (dal di dentro) da parte del dogmatico -il quale però, come si è visto, non è certo che disponga di un proprio concetto di norma. Tale concetto è esplicitato al livello della teoria del diritto, tuttavia non come concetto esplicativo e osservativo oppure ermeneutico, bensì come un postulato primitivo dal carattere meramente stipulativo-dunque addirittura ultraesterno rispetto alla pratica o alla realtà del diritto.

Il concetto di norma -leggiamo in Principia juris- non è una nozione la cui definizione possa dipendere dall'uso che eventualmente si faccia d'esso in questo o quel contesto legislativo. Cioè, è un 
concetto teorico convenzionale. La definizione di un concetto e la giustificazione di una tesi della dogmatica esigono la lettura dei testi normativi che costituirebbero la base empirica della dogmatica medesima. Non avrebbe senso e sarebbe vano invece, secondo Ferrajoli, cercare in quei testi il significato o il fondamento di un concetto o di una tesi della teoria. Mentre il linguaggio della dogmatica, pur essendo osservativo e protocollare, rimane un linguaggio comune, il linguaggio della teoria del diritto è del tutto artificiale. La teoria qui non è solo un metalinguaggio, ma più specificamente è un metalinguaggio che non riproduce il linguaggio comune che ha per oggetto, che non si alimenta di questo ed anzi lo rigetta.

Il linguaggio giuridico difatti, sebbene si configuri come uno specifico linguaggio tecnico, rimane un linguaggio «comune», in tanto in quanto l'aggettivo «comune» si contrapponga agli aggettivi «convenzionale», «artificiale», o «formale». Un linguaggio comune come tale non è suscettibile di formalizzazione, potendo essere invece analizzato, come accade nelle discipline giuridiche positive, in un metalinguaggio del pari comune, sebbene dotato di maggiore precisione e chiarezza. La teoria del diritto, al contrario, essendo elaborata in un linguaggio interamente artificiale e convenzionale, è un sistema di concetti ed enunciati che può essere perfettamente organizzato secondo una struttura formale, rigorosa ed esattamente prestabilita.

In questa prospettiva, il linguaggio della teoria risulta non soltanto esterno alla pratica giuridica (che si dà nella produzione e interpretazione dei testi normativi), ma estremamente esterno. Non è un linguaggio, come quello di un eventuale spettatore, che spieghi il diritto dal punto di vista della fatticità delle norme e della regolarità della pratica giuridica, del suo tessuto di cause ed effetti. Risulta ora essere qualcosa di molto più esterno. In esso non vige più il primato della adaequatio intellectus ad rem, di una «verità giuridica»-se si vuole- che lo ancorerebbe bene o male ancora al linguaggio comune su cui verte. Il principio che qui ora sembra vigere è la pretesa del teorico di riformare e purificare -sia pure, si afferma, a fini esplicativi- ciò che gli appare gravemente confuso, incoerente ed indeterminato. È piuttosto il principio della adaequatio rei ad intellectum, di una verità ideale che produce la cosa reale, punto di vista esterno quanto nessun altro.

Ciò è reso ancora più evidente dalla successiva articolazione della teoria del diritto secondo il metodo assiomatico. Qui si pongono sedici termini primitivi, tra cui vale menzionare la presenza per esempio di «democratico». Si pongono poi una serie di «postulati» tra i quali può menzionarsi come particolarmente significativo quello per cui «i soggetti non sono oggetti», il quale corrisponde grosso modo alla seconda formulazione dell'imperativo categorico kantiano. Si tratta in entrambi i casi (nel caso del termine «democratico» e del postulato «i soggetti non sono oggetti») di concetti la cui complessità e il cui carattere apprezzativo, normativo forte, rendono genuino e ragionevole l'eventuale disaccordo rispetto al loro contenuto. Possono ritenersi 
dunque concetti «essenzialmente controversi» ${ }^{49}$. La loro controvertibilità consiglierebbe per una loro sostanziosa ed abbondante giustificazione e dovrebbe indurre a sostenerne ed a provarne la dimostrabilità, sia pure argomentativa. Si tratta di concetti tanto «spessi» che risulta implausibile ed imprudente presupporne l'indimostrabilità discorsiva e assumerne l'evidenza intuitiva.

D'altra parte, tale loro controvertibilità e questo loro «spessore» (e la loro «profondità» storica potrebbe aggiungersi) si sottraggono facilmente allo sforzo di porre condizioni necessarie e sufficienti del loro uso corretto. La regola del loro uso non si lascia determinare in termini puramente semantici o «criteriali». Qui -come direbbe Wittgenstein- l'esempio illustra la regola e la riarticola, e il principio poi, il «succo», che sta alla base delle regole e della loro prassi, o del «gioco linguistico» entro cui si dà, ci guida nell' attribuzione di significato alla regola. Non ogni significato ascrivibile alla regola è quello corretto, ma solo il significato ragionevole ${ }^{50}$.

È vero -ci dice Ferrajoli-, nel caso dei concetti della teoria del diritto si tratta di nozioni che quasi tutte hanno una tradizione millenaria nel corso della quale sono state impiegate nei modi e significati più disparati, a volta persino all'interno dell'opera di un medesimo autore, dando origine frequentemente a una serie di pseudoproblemi. Ebbene -dichiara allora programmaticamente Ferrajoli- di questa vetusta, antica tradizione, di tali usi storici la teoria del diritto è chiamata a fare tabula rasa.

È significativo a questo proposito il ruolo che Ferrajoli attribuisce alla logica del diritto. Questa opera come somma di principia juris tantum, come operazione solo del teorico, e non già come principia juris et in jure, come somma cioè di criteri che indirizzino anche il discorso giuridico pratico. La logica-leggiamo nell'introduzione al primo volume di Principia juris- è propria di discorsi sul diritto, di metadiscorsi, ma non lo è del discorso di primo grado del diritto positivo, rispetto al quale di conseguenza essa (la logica) è detta svolgere un ruolo non già descrittivo o ricostruttivo, bensì rigidamente prescrittivo. Mutatis mutandis, sarebbe come dire -mi sembra- che la grammatica e le sue regole non sono proprie di un certo linguaggio naturale, bensì solo del discorso dei grammatici i quali esercitano una funzione imperativa e disciplinare su quel certo linguaggio naturale.

Una definizione deve stipulare con rigore le condizioni tanto necessarie quanto sufficienti per l'uso del termine definito. Ciò ci con-

49 Su questi cfr. l'ormai classico contributo di Walter B. Gallie, Essentially Contested Concepts, in «Proceedings of the Aristotelian Society», vol. 56, 1956, pp. 167-198.

${ }_{50}$ Cfr. quanto scrive Hilary Putnam: «Chi non afferra il "succo" del gioco linguistico, e non riesce a porsi con l'immaginazione nella posizione di una persona impegnata nel gioco, non può valutare se in esso i "criteri" vengono applicati ragionevolmente o no» (H. Putnam, Il pragmatismo: una questione aperta, trad. it. di M. Dell'Utri, Laterza, Roma-Bari 2003, p. 56, corsivo nel testo). 
sentirà -questa è l'accattivante promessa di Ferrajoli- di costruire e sviluppare con rigore un sistema trasparente, e soprattutto coerente e non contraddittorio di tesi.

Tuttavia, la controvertibilità dei termini primitivi e dei postulati non si risolve mediante la mera stipulazione. L'univocità non riesce qui ad ottenersi con un atto decisionistico, o con una scelta monologica del teorico. Tale scelta, in assenza di un rinvio agli usi della pratica su cui interviene metalinguisticamente e altresì in assenza di una giustificata e sostanziale motivazione, appare tutt'altro che trasparente. Certo, sappiamo chi è l'autore della scelta, ma il suo sostrato materiale e la teoria normativa retrostante rimangono nondimeno oscuri, non sono resi pubblici. L'oscurità e la mancanza di pubblicità sono poi rafforzate dall'opzione metaetica noncognitivistica dell'autore, che non ci permette di ripercorrere e di controllare i passaggi argomentativi mediante i quali egli giunge alle sue stipulazioni valorative.

Scelte tanto foriere di successivi sviluppi meriterebbero d'essere tematizzate e per così dire rielaborate (e rese condivisibili) attraverso un lavoro di ricognizione e di argomentazione delle concezioni e delle tesi che si sovrappongono e rincorrono per la definizione e l'uso dei concetti in gioco, ponderandone le ulteriori implicazioni normative. L'assunzione dell'indimostrabilità, nel caso di postulati e termini che sono tutt'altro che intuitivi o «chiari e distinti», ne mantiene intatta la (ragionevole) controvertibilità e non garantisce, anzi impedisce infine, che nessun postulato o nessuna tesi, sia pure derivata o derivabile con tutto il rigore logico possibile, non risulti nondimeno la negazione di qualcuno dei postulati medesimi. Insomma, data l'ignorata, ma latente contestabilità di termini e postulati, non può escludersi che la teoria che da questi muove, per quanto formale e rigorosa, non contenga simultaneamente una tesi vera ed una tesi falsa.

Fecha de recepción: 30/06/2012. Fecha de aceptación: 8/11/2012. 